\title{
EFEITOS DA CINESIOTERAPIA SOBRE A FORÇA DE PREENSÃO PALMAR EM INDIVÍDUOS COM DOENÇAS REUMÁTICAS
}

\author{
EFFECTS OF KINESIOTHERAPY ON PALMAR GRIP STRENGTH IN INDIVIDUALS
}

\author{
WITH RHEUMATIC DISEASES
}

\author{
Matheus Santos Gomes Jorge ${ }^{1}$ \\ Willian Guerra de Lima² \\ Patrícia Rodigheri Vieira ${ }^{3}$ \\ Letícia Antoniolli Siiss ${ }^{4}$ \\ Caroline Zanin ${ }^{5}$ \\ Sabrina Casarin Vogelmann 6 \\ Lia Mara Wibelinger ${ }^{7}$ \\ Luciane Daroit ${ }^{8}$
}

Recebido em: 31 jul. 2017

Aceito em: 24 abr. 2018

RESUMO: Introdução: as doenças reumáticas apresentam manifestações musculoesqueléticas e sistêmicas que podem acometer quaisquer regiões do corpo. No caso das mãos, uma das manifestações é a diminuição da força de preensão palmar destes indivíduos. Objetivo: verificar os efeitos da cinesioterapia sobre a força de preensão palmar em indivíduos com doenças reumáticas. Material e métodos: estudo longitudinal e intervencionista com 24 indivíduos portadores de doenças reumáticas (osteoartrite, artrite reumatoide, fibromialgia, lúpus eritematoso sistêmico, esclerose sistêmica e dermatopolimiosite), com idade média de 50,23 anos. Os indivíduos realizaram 10 sessões de fisioterapia, baseadas em cinesioterapia, com exercícios globais e funcionais, 02 vezes por semana, com duração média de 50 minutos, de março de 2014 a novembro de 2015, na Clínica de Fisioterapia da Universidade de Passo Fundo, Passo Fundo/RS. As avaliações inicial e final envolveram a coleta de dados e a mensuração da força de preensão palmar, por meio da dinamometria manual. Resultados: observou-se que os indivíduos apresentaram melhora da força de preensão palmar, porém os resultados foram estatisticamente significativos apenas para a mão direita dos indivíduos com osteoartrite, para ambas as mãos dos indivíduos com artrite reumatoide e lúpus eritematoso sistêmico

\footnotetext{
1 Fisioterapeuta, Pós-graduando do Curso de Especialização em Fisioterapia Traumato-ortopédica e mestrando do Programa de Pós-graduação em Envelhecimento Humano, Universidade de Passo Fundo.

${ }^{2}$ Graduando do Curso de Fisioterapia, Faculdade de Educação Física e Fisioterapia, Universidade de Passo Fundo, bolsista Pibic/CNPq.

${ }^{3}$ Graduanda do Curso de Fisioterapia, Faculdade de Educação Física e Fisioterapia, Universidade de Passo Fundo, bolsista Pibic/UPF.

${ }^{4}$ Graduanda do Curso de Fisioterapia, Faculdade de Educação Física e Fisioterapia, Universidade de Passo Fundo.

${ }^{5}$ Graduanda do Curso de Fisioterapia, Faculdade de Educação Física e Fisioterapia, Universidade de Passo

Fundo.

${ }^{6}$ Graduanda do Curso de Fisioterapia, Faculdade de Educação Física e Fisioterapia, Universidade de Passo Fundo, bolsista Probic/Fapergs.

7 Doutora em Gerontologia Biomédica pela Pontifícia Universidade Católica/RS. Docente do Curso de Fisioterapia e do Programa de Pós-Graduação em Envelhecimento Humano, Faculdade de Educação Física e Fisioterapia, Universidade de Passo Fundo.

8 Docente do Curso de Matemática, Instituto de Ciências Exatas e Geociências, Universidade de Passo Fundo.
} 
e para a força de preensão palmar geral dos indivíduos. Conclusão: o protocolo fisioterapêutico proposto produziu aumento da força de preensão palmar de indivíduos com doenças reumáticas.

Palavras-chave: Força da mão. Doenças reumáticas. Fisioterapia. Reabilitação. Exercício.

ABSTRACT: Introduction: rheumatic diseases present musculoskeletal and systemic manifestations that can affect any region of the body. In the case of the hands, one of the manifestations is the decrease of the handgrip strength of these individuals. Aim: to verify the effects of kinesiotherapy on handgrip strength in individuals with rheumatic diseases. Material and methods: longitudinal and interventional study with 24 individuals with rheumatic diseases (osteoarthritis, rheumatoid arthritis, fibromyalgia, systemic lupus erythematosus, systemic sclerosis and dermatopolymyositis), mean age 50.23 years. The individuals performed 10 sessions of physiotherapy, based on kinesiotherapy, with global and functional exercises, 02 times a week, with an mean duration of 50 minutes, from March 2014 to November 2015, at the Physiotherapy Clinic of Passo Fundo University, Passo Fundo/RS. The initial and final evaluations involved data collection and measurement of handgrip strength using manual dynamometry. Results: it was observed that the individuals presented improvement of the handgrip strength, but the results were statistically significant only for the right hand of individuals with osteoarthritis, for both hands of individuals with rheumatoid arthritis and systemic lupus erythematosus and for the handgrip strength of individuals. Conclusion: the proposed physiotherapeutic protocol produced an increase in the handgrip strength of individuals with rheumatic diseases.

Keywords: Hand strength. Rheumatic diseases. Physical therapy specialty. Rehabilitation. Exercise.

\section{INTRODUÇÃO}

As doenças reumáticas (DR) são um grupo de patologias que podem manifestarse de forma aguda, recorrente ou crônica. Atingem todas as faixas etárias, são caracterizadas por incapacidade funcional e podem gerar altos custos com a saúde, por meio de consultas médicas, medicamentos, reabilitação ou gastos indiretos (PORTUGAL, 2013). De acordo com a literatura, no Brasil, as DR mais incidentes são a osteoartrite, a fibromialgia, a artrite reumatoide e o lúpus eritematoso sistêmico, sendo que acometem preferencialmente o gênero feminino entre os 35 e os 40 anos (CARVALHO; COSTA; SILVA, 2013).

Dentre os segmentos corporais de suma necessidade para a independência nas atividades de vida diária se encontra a mão, um dos principais instrumentos do corpo humano, especialmente em relação a sua peculiaridade de força de preensão palmar (FPP) (DIAS et al., 2010). As DR, por sua vez, podem acometer os órgãos e os sistemas, danificando as articulações e gerando deformidades nas extremidades. Desta forma, as mãos podem ser acometidas por tais patologias, gerando diversas limitações que podem evoluir para lesões cutâneas (KALUGA et al., 2014).

Atualmente, há um consenso de diretrizes internacionais, recomendações de sociedades científicas e avaliações estruturadas de que o exercício físico deva ser prescrito 
para indivíduos com DR, especialmente a cinesioterapia (MADDALI BONGI; DEL ROSSO). Isso se deve, ao possível efeito anti-inflamatório do exercício físico regular sobre essas e sua potencial capacidade de reduzir o consumo e/ou as doses das drogas imunossupressoras. Assim sendo, os objetivos consistem em atenuar os sintomas causados pelas DR, melhorar a amplitude de movimento, a força muscular, o bem estar físico e, consequentemente, melhorar sua qualidade de vida (GUALANO et al., 2011).

Neste sentido, o presente estudo teve como objetivo verificar os efeitos da cinesioterapia sobre a FPP de indivíduos com DR.

\section{MATERIAL E MÉTODOS}

Trata-se de um estudo longitudinal e intervencionista que faz parte de um projeto guarda-chuva denominado "Efeitos do tratamento fisioterapêutico em pacientes portadores de doenças reumáticas", aprovado pelo Comitê de Ética em Pesquisa em Seres Humanos da Universidade de Passo Fundo sob protocolo nํㅜ 348.381 e está de acordo com resolução 466/2012 do Conselho Nacional de Saúde e com a Declaração de Helsinki de 1975, revisada em 2000 .

Inicialmente, foram recrutados para o estudo 24 indivíduos com diagnóstico clínico de alguma DR, por meio de uma amostragem não probabilística, por conveniência. Todos estavam em uma lista de espera para atendimento fisioterapêutico na Clínica de Fisioterapia da Universidade de Passo Fundo.

\section{CRITÉRIOS DE INCLUSÃO E EXCLUSÃO}

Os critérios de inclusão para o estudo eram indivíduos com diagnóstico clínico de alguma DR, com idade maior ou igual a 18 anos, capazes de compreender a dinâmica dos exercícios, que não estivessem realizando tratamento fisioterapêutico prévio por pelo menos 03 meses. Mediante isso, seriam excluídos do estudo atual os indivíduos que apresentassem impossibilidade física ou mental de realizar os testes solicitados, tais como amputação do membro superior ou quadro demencial associado, indivíduos com próteses em alguma articulação do membro superior e indivíduos que não completassem todas as sessões do tratamento proposto.

Todos os indivíduos concordaram em participar do estudo e assinatura o Termo de Consentimento Livre e Esclarecido. Após foram submetidos a uma avaliação inicial e final, bem como a um programa de intervenção fisioterapêutico.

\section{PROCEDIMENTO DE COLETA DE DADOS}

A avaliação pré- intervenção fisioterapêutica envolveu uma coleta de dados e 
avaliação da FPP. A coleta de dados foi realizada por meio de um questionário sociodemográfico elaborado pelos próprios autores, contendo informações tais como, gênero, idade, histórico da doença atual, diagnóstico médico, entre outros.

A avaliação da FPP foi realizada por meio da dinamometria manual, utilizando-se o dinamômetro da marca Kratos $\AA$, cujo mesmo é um sistema hidráulico fechado, constituído por alças fixas inadaptáveis, onde aplica-se uma contração isométrica, registrada em quilogramas força (kgf), e com o visor de leitura voltado para o indivíduo permitindo-o acompanhar seu desempenho no teste (MYRA et al., 2015). O indivíduo deveria estar sentado, com cotovelo flexionado a $90^{\circ}$ e antebraço paralelo ao chão, e aplicar uma contração isométrica máxima durante três segundos em três tentativas. Após seria realizada a média aritmética das três tentativas de cada membro (STOCKTON et al., 2011). Quanto maior o valor obtido, maior é a força de preensão palmar do indivíduo.

\section{PROGRAMA DE INTERVENÇÃO}

As sessões de fisioterapia foram realizadas na Clínica de Fisioterapia da Faculdade de Educação Física e Fisioterapia, da Universidade de Passo Fundo, Passo Fundo/RS, entre os meses de março de 2014 a novembro de 2015. Os indivíduos realizaram 10 sessões interventivas, 02 vezes por semana, com duração média de 50 minutos. 0 protocolo fisioterapêutico sancionado foi a cinesioterapia, e os exercícios globais e funcionais, realizados em solo, foram delineados na seguinte ordem:

- Exercícios respiratórios com padrão diafragmático em posição sentada. Com as mãos na barriga, deveriam inspirar pelo nariz, enquanto realizavam a expansão do abdômen e, após, deveriam expirar pela boca, enquanto realizavam a contração do abdômen. Foram realizados cinco ciclos inspiratórios em três repetições;

- Mobilizações articulares passivas de punhos, metacarpos e falanges das mãos, com os indivíduos sentados e membro superior apoiado sobre uma mesa;

- Fortalecimento dos músculos flexores de ombro com os indivíduos sentados em uma bola suíça e segurando um bastão sem carga, progredindo até $02 \mathrm{~kg}$, associado à rotação lateral de tronco (03 séries de 10 repetições);

- Fortalecimento de bíceps braquial com halter de $500 \mathrm{~g}$, progredindo até $02 \mathrm{~kg}$, e com os indivíduos em pé (03 séries de 10 repetições);

- Fortalecimento dos músculos extensores de ombro com faixa elástica rosa, progredindo até a faixa elástica azul (03 séries de 10 repetições);

- Fortalecimento de músculos rotadores internos e externos de ombro com faixa elástica rosa, progredindo até a cor verde (03 séries de 10 repetições);

- Fortalecimento dos músculos abdutores de ombro com halter $500 \mathrm{~g}$, progredindo até $01 \mathrm{~kg}$, e os indivíduos em pé (03 séries de 10 repetições); 
- Fortalecimento dos membros superiores por meio das diagonais do método Facilitação Neuromuscular Proprioceptiva com faixa elástica rosa, progredindo até a faixa elástica cinza (03 séries de 10 repetições);

- Exercícios para as mãos: fortalecimento de punhos com halter 500g, progredindo até $1 \mathrm{~kg}$ (02 séries de 10 repetições); fortalecimento de dedos com exercitador de mãos e dedos da cor "vermelha carga 1.4", progredindo até a cor "preta 4.1", e fortalecedor de dedos em formato de rede (1 série de 10 repetições); fortalecimento de mãos com bolinhas proprioceptivas "suaves", progredindo até a carga "média" (02 séries de 10 repetições); exercícios de motricidade fina com brinquedos de peças móveis.

- Exercícios de equilíbrio, propriocepção e transferência de peso no balance pad, balance pad em formato de disco, cama elástica, balancim e pranchas de Freeman, progredindo do menos complexo até o mais complexo;

- Exercício de marcha em rampas e escadas inicialmente sem obstáculos, progredindo para com obstáculos (cones, rolos e bolas) durante o percurso;

- Pompagens das regiões cervical, escapular, peitoral e sacral; desativação de tender points na região escapular e espinhal com o indivíduo deitado em uma maca variando nas posições de decúbito dorsal ou ventral;

- Alongamentos ativos globais no final da sessão dos principais grupos musculares dos membros superiores e inferiores e de tronco (15 segundos cada grupo muscular).

A troca de dispositivo ou da carga dos equipamentos foi determinada conforme a capacidade de cada indivíduo e obedeceu a uma contínua progressão, sendo do mais leve ou fácil até o mais pesado ou difícil. Os indivíduos foram orientados a interromper a atividade para descanso quando sentissem dificuldade para concluir as séries propostas e adotou-se intervalo de 30 segundos a 1 minuto entre estas.

Após as 15 sessões de intervenção fisioterapêutica todos os parâmetros foram reavaliados.

\section{ANÁLISE ESTATÍSTICA}

Para a análise estatística foi utilizado o programa Microsoft Excel 2010 e o programa Wilcoxom. A estatística descritiva foi utilizada para caracterizar a amostra do estudo atual o teste $t$ de Student de amostras pareadas foi utilizado para analisar as variáveis pré e pós intervenção, considerando uma significância de $p \leq 0,05$.

\section{RESULTADOS E DISCUSSÃO}

Todos os indivíduos inicialmente recrutados preencheram os critérios de inclusão 
deste estudo e realizaram todas as sessões de fisioterapia propostas. A idade média dos participantes foi de $\pm 50,23$ anos.

\section{A tabela 1 apresenta os dados da caracterização da amostra estudada.}

Tabela 1: Caracterização do perfil socioeconômico e das condições de saúde da amostra estudada

\begin{tabular}{|c|c|c|}
\hline \multirow[b]{2}{*}{ Variáveis } & \multicolumn{2}{|c|}{ Representaçao } \\
\hline & $\mathbf{N}$ & (\%) \\
\hline \multicolumn{3}{|l|}{ Diagnósticos clínicos } \\
\hline Osteoartrite & 06 & 25,0 \\
\hline Artrite reumatoide & 05 & 20,83 \\
\hline Fibromialgia & 04 & 16,66 \\
\hline Lúpus eritematoso sistêmico & 03 & 12,5 \\
\hline Esclerose sistêmica & 03 & 12,5 \\
\hline Dermatopolimiosite & 03 & 12,5 \\
\hline \multicolumn{3}{|l|}{ Tempo de diagnóstico } \\
\hline$\leq 04$ anos & 04 & 16,7 \\
\hline 05-09 anos & 04 & 16,7 \\
\hline 10-14 anos & 08 & 33,3 \\
\hline$\geq 15$ anos & 08 & 33,3 \\
\hline \multicolumn{3}{|l|}{ Gênero } \\
\hline Feminino & 21 & 87,5 \\
\hline Masculino & 03 & 12,5 \\
\hline \multicolumn{3}{|l|}{ Faixa etária } \\
\hline$\leq 39$ anos & 02 & 8,3 \\
\hline 40-49 anos & 06 & 25,0 \\
\hline $50-59$ anos & 08 & 33,3 \\
\hline 60-69 anos & 03 & 12,5 \\
\hline$\geq 70$ anos & 05 & 20,8 \\
\hline \multicolumn{3}{|l|}{ Escolaridade } \\
\hline Ensino fundamental incompleto ou completo & 08 & 33,33 \\
\hline Ensino médio incompleto ou completo & 14 & 58,53 \\
\hline Ensino superior incompleto ou completo & 02 & 8,33 \\
\hline \multicolumn{3}{|l|}{ Estado civil } \\
\hline Casados & 06 & 25,0 \\
\hline Solteiros & 12 & 50,0 \\
\hline Viúvo & 05 & 20,83 \\
\hline Separados / Divorciados & 01 & 4,16 \\
\hline \multicolumn{3}{|l|}{ Filhos } \\
\hline Nenhum & 09 & 37,5 \\
\hline 01 filho & 02 & 8,33 \\
\hline 02 filhos & 08 & 33,33 \\
\hline 03 filhos ou mais & 05 & 20,83 \\
\hline \multicolumn{3}{|l|}{ Queixa principal } \\
\hline Dor & 19 & 79,16 \\
\hline Fraqueza muscular & 04 & 16,66 \\
\hline Perda de equilíbrio & 01 & 4,16 \\
\hline \multicolumn{3}{|l|}{ Uso de medicamentos } \\
\hline Sim & 24 & 100 \\
\hline Não & - & - \\
\hline \multicolumn{3}{|l|}{ Classes medicamentas } \\
\hline Anti-inflamatório & 10 & 41,66 \\
\hline Hipotensores & 10 & 41,66 \\
\hline Analgésicos & 09 & 37,5 \\
\hline Repositores vitamínicos & 07 & 29,16 \\
\hline Outros & 13 & 54,16 \\
\hline \multicolumn{3}{|l|}{ Doenças associadas } \\
\hline Sim & 16 & 66,66 \\
\hline Não & 08 & 33,33 \\
\hline
\end{tabular}




\begin{tabular}{lcc} 
& \multicolumn{2}{c}{ Representação } \\
\cline { 2 - 3 } Variáveis & $\mathbf{N}$ & $(\%)$ \\
\hline Tipos de doenças associadas & & \\
\hline Hipertensão arterial sistêmica & 08 & 33,33 \\
Outra(s) doença(s) reumática(s) & 06 & 25,0 \\
Hipotireoidismo & 04 & 16,66 \\
Diabetes mellitus & 04 & 16,66 \\
Doença cardiovascular & 02 & 8,33 \\
Outras & 05 & 20,83 \\
\hline Histórico familiar de doença reumática & & \\
\hline Sim & 30 & 57,0 \\
Não & 22 & 42,0 \\
\hline
\end{tabular}

Legenda: $\mathrm{n}=$ valor absoluto; $\%$ = valor relativo

Em linhas gerais, as DR mais predominantes foram a osteoartrite (25\%) e a artrite reumatoide $(20,83 \%)$. Observou-se que a amostra era predominantemente do gênero feminino (87,5\%), concentrada na faixa etária dos 50 aos 59 anos (33,3\%). A maioria dos indivíduos possuía diagnóstico da DR há 10 anos ou mais $(66,6 \%)$, possuía escolaridade básica $(91,66 \%)$, possuía pelo menos dois filhos $(33,33 \%)$ e eram solteiros $(50 \%)$. Apresentavam como queixa principal algum tipo de dor $(79,16 \%)$. Todos faziam uso de medicamentos contínuos, sendo os anti-inflamatórios $(41,66 \%)$ e os hipotensores $(41,66 \%)$ os principais tipos. A maioria possuía doenças associadas $(66,66 \%)$ e sendo a hipertensão arterial sistêmica a mais recorrente (33,33\%). E, por fim, a maioria apresentava histórico familiar de DR (54,16\%).

A tabela 2 apresenta os dados relativos à FPP da mão direita nos indivíduos do estudo atual nas fases pré e pós intervenção fisioterapêutica.

Tabela 2: Força de preensão palmar da mão direita pré e pós intervenção fisioterapêutica

\begin{tabular}{llll}
\hline Tipo de doença reumática & $\begin{array}{l}\text { Pré intervenção (média } \\
\mathbf{\pm} \text { DP) }\end{array}$ & $\begin{array}{l}\text { Pós intervenção } \\
\text { (média } \pm \text { DP) }\end{array}$ & $\begin{array}{l}\text { Valor de } \mathbf{p} \\
(\leq \mathbf{0}, \mathbf{0 5})\end{array}$ \\
\hline Osteoartrite & $13,2 \mathrm{kgf} \pm 0,68$ & $16,5 \mathrm{kgf} \pm 0,52$ & $0,05^{*}$ \\
Artrite Reumatoide & $4,4 \mathrm{kgf} \pm 0,24$ & $15,9 \mathrm{kgf} \pm 0,75$ & $0,02^{*}$ \\
Fibromialgia & $13,9 \mathrm{kgf} \pm 0,86$ & $17,6 \mathrm{kgf} \pm 1,16$ & 0,11 \\
Lúpus eritematoso sistêmico & $3,6 \mathrm{kgf} \pm 0,23$ & $16,5 \mathrm{kgf} \pm 0,60$ & $0,05^{\star}$ \\
Esclerose sistêmica & $14,0 \mathrm{kgf} \pm 0,87$ & $13,1 \mathrm{kgf} \pm 1,06$ & 0,94 \\
Dermatopolimiosite & $4,3 \mathrm{kgf} \pm 0,49$ & $21,0 \mathrm{kgf} \pm 1,34$ & 0,11 \\
\hline
\end{tabular}

Legenda: $\mathrm{DP}=$ desvio padrão; $\mathrm{kgf}$ = quilograma-força; ${ }^{*}$ = estatisticamente significativo

Notou-se que, após a intervenção fisioterapêutica, a FPP da mão direita dos indivíduos com osteoartrite, artrite reumatoide e lúpus eritematoso sistêmico apresentaram resultados estatisticamente significativos $(p \leq 0,05)$.

A tabela 3 apresenta os dados relativos à FPP da mão esquerda nos indivíduos do estudo atual nas fases pré e pós intervenção fisioterapêutica. 
Tabela 3: Força de preensão palmar da mão esquerda pré e pós intervenção fisioterapêutica

\begin{tabular}{llll}
\hline Tipo de doença reumática & $\begin{array}{l}\text { Pré intervenção (média } \\
\mathbf{\pm} \mathbf{D P})\end{array}$ & $\begin{array}{l}\text { Pós intervenção } \\
\text { (média } \pm \text { DP) }\end{array}$ & $\begin{array}{l}\text { Valor de } \mathbf{p} \\
(\leq \mathbf{0}, \mathbf{0 5})\end{array}$ \\
\hline Osteoartrite & $10,9 \mathrm{kgf} \pm 0,79$ & $15,6 \mathrm{kgf} \pm 0,44$ & 0,19 \\
Artrite Reumatoide & $5,6 \mathrm{kgf} \pm 0,28$ & $16,3 \mathrm{kgf} \pm 0,71$ & $0,01^{*}$ \\
Fibromialgia & $11,7 \mathrm{kgf} \pm 0,72$ & $14,8 \mathrm{kgf} \pm 0,98$ & 0,14 \\
Lúpus eritematoso sistêmico & $3,6 \mathrm{kgf} \pm 0,05$ & $16,8 \mathrm{kgf} \pm 0,24$ & $0,008^{*}$ \\
Esclerose sistêmica & $12,6 \mathrm{kgf} \pm 0,64$ & $18,1 \mathrm{kgf} \pm 0,43$ & 0,35 \\
Dermatopolimiosite & $4,6 \mathrm{kgf} \pm 0,37$ & $17,6 \mathrm{kgf} \pm 1,19$ & 0,16 \\
\hline
\end{tabular}

Legenda: DP = desvio padrão; kgf = quilograma-força; ${ }^{*}=$ estatisticamente significativo

Notou-se que, após a intervenção fisioterapêutica, a FPP da mão esquerda dos indivíduos com artrite reumatoide e lúpus eritematoso sistêmico apresentaram resultados estatisticamente significativos $(p \leq 0,05)$.

A tabela 4 apresenta os dados referentes a FPP geral das mãos direita e esquerda dos indivíduos nas fases pré e pós intervenção fisioterapêutica.

Tabela 4: Força de preensão palmar geral pré e pós intervenção fisioterapêutica.

\begin{tabular}{llll}
\hline & $\begin{array}{l}\text { Pré intervenção } \\
\text { (média } \pm \text { DP) }\end{array}$ & $\begin{array}{l}\text { Pós intervenção } \\
\text { (média } \pm \text { DP) }\end{array}$ & $\begin{array}{l}\text { Valor de } \mathbf{p} \\
(\leq \mathbf{0}, \mathbf{0 5})\end{array}$ \\
\hline Mão direita & $9,3 \mathrm{kgf} \pm 0,73$ & $16,7 \mathrm{kgf} \pm 0,81$ & $0,001^{*}$ \\
Mão esquerda & $8,4 \mathrm{kgf} \pm 0,62$ & $16,3 \mathrm{kgf} \pm 0,64$ & $0,00001^{*}$ \\
\hline
\end{tabular}

Legenda: $\mathrm{DP}=$ desvio padrão; $\mathrm{kgf}=$ quilograma-força; ${ }^{*}=$ estatisticamente significativo

Após a intervenção fisioterapêutica, a FPP de ambas as mãos apresentaram melhora estatisticamente $(p \leq 0,05)$.

A mão tem uma função isométrica de preensão palmar com finalidade de prender algum objeto com os dedos parcialmente fletidos contra a palma, utilizando a contrapressão do polegar aduzido. Contrações musculares intrínsecas e extrínsecas ocorrem de forma conjunta durante este movimento permitindo uma estabilização da articulação do punho durantes os movimentos dos dedos sobre a mão que, desta forma, poderá realizar suas atividades de vida diária (DIAS et al., 2010; KISNER; COLBY; CAROMANO, 2009; CIMA et al., 2013).

Neste sentido, a avaliação da FPP torna-se uma medida válida e confiável realizada para comparar a eficácia de vários procedimentos, avaliar a funcionalidade dos indivíduos e definir metas para o tratamento (FIGUEIREDO et al., 2007), sendo esta testada por meio da dinamometria manual, um método capaz de avaliar a condição física dos membros superiores. Apresenta grande aplicabilidade, baixo custo, não invasividade e capacidade de detectar precocemente distúrbios metabólicos musculares que interfiram na capacidade dos músculos em produzir força (NOVAES et al., 2009). O que justifica a escolha deste método como forma de avaliação para os indivíduos com doenças reumáticas.

A mensuração da FPP pela dinamometria manual estima a força gerada pelos músculos flexores da mão e do antebraço. Este método torna-se um meio importante para acompanhar os seres humanos em diversas situações da vida, entre elas o surgimento de 
lesões e procedimentos de reabilitação (HOGREL, 2015). O que levou aos autores optar pela avaliação da FPP nos indivíduos com DR do estudo atual, onde foi possível observar déficits na fase pré intervenção fisioterapêutica e correções na fase pós intervenção.

A força muscular é equivalente ao número de sarcômeros que estão presentes no músculo a serem recrutados durante uma contração e a forma como se dispõe. Há vários fatores que podem influenciá-la, como a idade, o gênero, as características antopométricas e fatores fisiológicos do tecido muscular (alterações de temperatura, $\mathrm{pH}$, fluxo sanguíneo e acúmulo de produtos metabólicos celulares) (GUYTON; HALL, 2000; HEFFERNAN et al., 2012). Fatores que estes que podem ter influenciado nos resultados obtidos para análise dos indivíduos, onde algumas DR apresentaram melhora estatisticamente significativa na FPP e outras não.

Em condições patológicas como a osteoartrite a articulação entre o carpo e o polegar pode ser comprometida, resultando na degeneração das superfícies articulares e interferindo na funcionalidade das mãos e, logicamente, da FPP (LEE et al., 2012). Nestes indivíduos a FPP correlaciona-se proporcionalmente com a funcionalidade das mãos e a dinamometria manual parece ser um meio adequado e de alta confiabilidade para avaliar a esta medida (NUNES et al., 2012; VILLAFAÑE et al., 2015). Visto que a osteoartrite pode acometer as mãos, é que este estudo preocupou-se em analisar a FPP nestes indivíduos. Apesar de não terem sido encontrados na literatura estudos semelhantes ao nosso em indivíduos com osteoartrite, pode-se observar que houve melhora estatisticamente significativa na FPP da mão direita destes indivíduos.

$\mathrm{Na}$ artrite reumatoide a FPP pode ser impactada por meio da inflamação persistente que causa destruição da articulação, desequilíbrio ligamentar e muscular acarretando em um desuso do membro afetado (IOP et al., 2015). Ainda, a perda da função das mãos pode ocasionar limitações e restrições da participação dos indivíduos com artrite reumatoide nas atividades em geral, bem como impactar na sua qualidade de vida (ØSTERÅS; KJEKEN, 2015). Neste sentido, um estudo buscou verificar a FPP em mulheres com artrite reumatoide. $O$ grupo intervenção ( $n=09$ com a doença) e o grupo controle ( $n=10$ sem a doença) foram submetidos a avaliação da FPP por meio da dinamometria manual, onde constatou-se que o grupo intervenção apresentou como queixa principal a dor e diminuição da FPP em relação aos indivíduos sem a doença (IOP et al., 2015). O que concorda com este estudo, visto que a maioria dos indivíduos apresentou a dor como queixa principal e os indivíduos com artrite reumatoide apresentaram impacto na FPP, assim como os indivíduos com outras DR.

Um estudo com 490 indivíduos com artrite reumatoide, randomizou-os em grupo intervenção $(n=246)$ e grupo controle $(n=244)$. O grupo intervenção realizou exercícios de fortalecimento e alongamento para os punhos e para as mãos, enquanto o grupo controle realizou apenas cuidados habituais e ao final do estudo os indivíduos que realizaram a fisioterapia apresentaram melhora na funcionalidade da mão, de acordo com o Michigan Hand Outcome Questionnaire (WILLIAMS et al., 2015). O que vai ao encontro do estudo atual, pois observou-se que houve melhora estatisticamente significativa da FPP em ambas 
as mãos do grupo de indivíduos com artrite reumatoide após as sessões de fisioterapia.

Alguns estudos têm referenciado que indivíduos com fibromialgia apresentam impacto na FPP se comparados a indivíduos sem a doença (CARDOSO et al., 2011; APARICIO et al., 2011). Um estudo que avaliou a FPP em 31 mulheres (16 com fibromialgia e 15 sem fibromialgia) observou que os portadores da doença apresentavam FPP de 20,9 kgf na mão esquerda e de 22,7 kgf na mão direita, enquanto nos indivíduos saudáveis a FPP foi de 27,0 kgf na mão esquerda e de 26,8 kgf na mão direita (CARDOSO et al., 2011).

O outro estudo avaliou 125 mulheres que foram randomizadas em grupo com fibromialgia $(n=81)$ e grupo sem fibromialgia $(n=44)$. Verificou-se que a FPP apresentou níveis menores nas mulheres com FM em relação as não portadoras da doença. Ainda, notou-se que quanto maior a gravidade da doença, menores eram os níveis de FPP (APARICIO et al., 2011). Desta forma, pode-se imaginar que a fibromialgia possa interferir na FPP, pois interferem na força muscular e na funcionalidade das mãos. O que concorda com o presente estudo que verificou impacto na FPP dos indivíduos com fibromialgia na fase pré intervenção.

Assim como nos indivíduos com osteoartrite, não foram encontrados estudos que comparassem a FPP pré e pós intervenção fisioterapêutica. Porém, neste estudo, constatou-se que os indivíduos com fibromialgia não apresentaram melhora estatisticamente significativa na FPP após a intervenção fisioterapêutica.

Aproximadamente, $90 \%$ dos indivíduos com lúpus eritematoso sistêmico relatam frequentemente dores, edemas e derrames articulares com artrite não erosiva principalmente nas mãos. Os comprometimentos articulares periféricos podem impactar na funcionalidade e na qualidade de vida dos portadores de lúpus eritematoso sistêmico (MYRA et al., 2015; VIANNA; SIMÕES; INFORZATO, 2010; SOCIEDADE BRASILEIRA DE REUMATOLOGIA, 2011; BALSAMO, 2012). Fatores estes que poderiam desencadear um processo de impacto na FPP dos indivíduos com lúpus eritematoso sistêmico.

Com base nisto, um estudo de caso envolveu um indivíduo do gênero feminino (49 anos), portadora de lúpus eritematoso sistêmico e artrite reumatoide há 15 anos. Este foi submetido a 15 sessões de fisioterapia, baseadas em cinesioterapia, 02 vezes semanais, durante 03 meses. A intervenção objetivou o alívio da dor, a redução da rigidez, o aumento da amplitude de movimento e da FPP. Após a intervenção, o indivíduo apresentou aumento da FPP de 2,3kgf na mão direita para 7,5kgf e de 3,3kgf na mão esquerda para 6,5kgf (MYRA et al., 2015). O que vai ao encontro do presente estudo, cujo mesmo analisou a FPP em indivíduos com lúpus eritematoso sistêmico e artrite reumatoide, e baseou-se, também, em cinesioterapia. Ao final, houve melhora estatisticamente significativa na FPP em ambas as DR.

Concordando com os resultados obtidos neste estudo em relação aos indivíduos com lúpus eritematoso sistêmico, outros estudos de caso realizados com indivíduos com lúpus eritematoso sistêmico associado a outras DR também evidenciaram resultados positivos sobre a FPP. Ambos submeteram os indivíduos com lúpus eritematoso sistêmico 
a um programa de cinesioterapia e hidrocinesioterapia. Enquanto um (JORGE; COMIN; WIBELINGER, 2016) encontrou aumento da FPP em 1,56kgf para a mão esquerda e $1,09 \mathrm{kgf}$ para a mão direita, o outro (JORGE et al., 2017) encontrou aumento de 2,2kgf para a mão direita e 1,2kgf para a mão esquerda.

A esclerose sistêmica e a dermatopolimiosite são patologias de ordem reumática que, juntamente com o lúpus eritematoso sistêmico, formam o subgrupo das doenças do tecido conjuntivo. Estas patologias também podem apresentar envolvimento das mãos em suas manifestações clínicas (JORGE; SCHNORNBERGER; SANTOS, 2017).

A esclerose sistêmica tem como manifestação principal o Fenômeno de Raynoud. Esta é a característica mais comum e a primeira a surgir, acometendo as extremidades como as mãos e os pés. Ele intensifica-se com situações de estresse e de frio, produzindo agravos como isquemias e ulcerações digitais e necrose tecidual. Ainda, estes indivíduos apresentam deficiência de vitamina $\mathrm{D}$, o que pode interferir em aspectos como a qualidade de vida e a capacidade de produzir uma FPP de boa qualidade (JORGE; SCHNORNBERGER; SANTOS, 2017; KAYSER; CORRÊA; ANDRADE, 2009; MACHADO; SOUTO; FREIRE, 2014; SAMPAIO-BARROS et al., 2016). Neste estudo os sujeitos com esclerose sistêmica não demonstraram melhora estatisticamente significativa nas avaliações pré e pós intervenção, possivelmente em virtude dos períodos de exacerbação do Fenômeno de Raynoud apresentado por estes.

Os resultados obtidos neste estudo com relação a FPP dos indivíduos com esclerose sistêmica, divergem dos resultados encontrados em um estudo de caso realizado com um idoso longevo portador de esclerose sistêmica. Os autores submeteram o indivíduo a 15 sessões de cinesioterapia e demonstraram aumento da FPP da mão direita em 11,1kgf e da mão esquerda em 19 kgf (DeMARCO et al., 2017).

$\mathrm{Na}$ dermatopolimiosite o principal sintoma é a fraqueza muscular. Além desta, os indivíduos podem apresentar acometimentos sistêmicos, tais como artrite simétrica não erosiva, Fenômeno de Raynaud e acometimento das mãos. As mãos adquirem aparência de "mãos de mecânico", caracterizado por descamações, fissuras, ceratose e hiperpigmentação simétrica e não pruriginosas nas palmas. Neste sentido, a avaliação dos indivíduos com dermatopolimiosite deve envolver testes específicos, como a dinamometria manual (DOURMISHEV; DOURMISHEV; SCHWARTZ, 2002; BABIĆ-NAGLIĆ, 2012; BARROS et al., 2014). É observado que as DR diminuem a funcionalidade do segmento afetado, entretanto os resultados dos indivíduos com dermatopolimiosite não foram estaticamente significativos após a intervenção fisioterapêutica proposta, pois a perda de força muscular torna-se um agravante que pode contribuir para o impacto da FPP nestes indivíduos.

Assim como em outras doenças já citadas neste artigo, não foram encontrados estudos que envolvessem o tratamento fisioterapêutico específico para as mãos em indivíduos com dermatopolimiosite.

Algumas limitações podem ser observadas no estudo atual. Uma delas é a carência 
literária em estudos semelhantes ao nosso, dificultando a estratégia de elaboração para um protocolo fisioterapêutico específico para a FPP de indivíduos com DR. Outro ponto que pode limitar o presente estudo, seria a falta de um grupo controle sem DR para comparar com os dados coletados. Embora o tamanho amostral seja restrito, este estudo abre a possibilidade para novas discussões de abordagens fisioterapêuticas que sejam eficazes para a melhora estatisticamente significativa da FPP em indivíduos com diferentes DR.

\section{CONSIDERAÇÕES FINAIS}

Em suma, o programa fisioterapêutico proposto foi benéfico para a melhora da FPP da mão direita nos indivíduos com osteoartrite, da FPP bilateral dos indivíduos com artrite reumatoide e lúpus eritematoso sistêmico. Além disso, houve melhora estatisticamente significativa na FPP geral bilateral dos indivíduos com DR.

\section{REFERÊNCIAS}

APARICIO, V. A. et al. Handgrip strength test as a complementary tool in the assessment of fibromyalgia severity in women. Archives of Physical Medicine and Rehabilitation, Philadelphia, v. 92, n. 1, p. 83-88, 2011.

BABIĆ-NAGLIĆ, D. Exercises in patients with myositis-active treatment intervention?

Reumatizam, Milão, v. 59, n. 2, p. 73-76, 2012.

BALSAMO, S. Análise comparativa da aptidão física de mulheres com lúpus eritematoso sistêmico. 2012. 107f. Tese (Doutorado em Ciências Médicas) Universidade de Brasília, Brasília.

BARROS, T. B. M. et al. Nefropatia por IgA e polimiosite: uma rara associação. Revista Brasileira de Reumatologia, Campinas, v. 54, n. 3, p. 231-233, 2014.

CARDOSO, F. S. et al. Avaliação da qualidade de vida, força muscular e capacidade funcional em mulheres com fibromialgia. Revista Brasileira de Reumatologia, Campinas, v. 51, n. 4, p. 344-350, 2011.

CARVALHO, F. M.; COSTA, M. C.; SILVA, T. C. D. Doenças reumáticas no Brasil: revisão de estudos epidemiológicos. Lecturas Educación Física y Deportes, Buenos Aires, v. 18, n. 184, p. 1, 2013.

CIMA, S. R. et al. Strengthening exercises to improve hand strength and functionality in rheumatoid arthritis with hand deformities: a randomized, controlled trial. Rheumatology International, Berlin, v. 33, n. 1, p. 725-732, 2013.

DeMARCO, M. et al. Efeitos da cinesioterapia sobre a força de preensão palmar e a qualidade de vida de um idoso longevo com esclerose sistêmica: relato de caso. Revista de Terapia Ocupacional da Universidade de São Paulo, São Paulo, v. 28, n. 1, p. 128134, 2017. 
DIAS, A. J. et al. Força de preensão palmar: métodos de avaliação e fatores que influenciam a medida. Revista Brasileira de Cineantropometria e Desempenho Humano, Florianópolis, v. 12, n. 3, p. 209-216, 2010.

DOURMISHEV, L. A.; DOURMISHEV, A. L.; SCHWARTZ, R. A. Dermatomyositis: cutaneous manifestations of its variants. International Journal of Dermatology, Philadelphia, v. 41, n. 1, p. 625-630, 2002.

FIGUEIREDO, I. M. et al. Teste de força de preensão utilizando o dinamômetro Jamar. Acta Fisiatrica, São Paulo, v. 14, n. 2, p. 104-110, 2007.

GUALANO, B. et al. Therapeutic effects of exercise training in patients with pediatric rheumatic diseases. Revista Brasileira de Reumatologia, Campinas, v. 51, n. 5, p. 490496, 2011.

GUYTON, A. C.; HALL, J. E. Textbook of Medical Physiology. 10th ed. Philadelphia: Saunders Elsevier, 2000.

HEFFERNAN, K. S. et al. Systemic vascular function is associated with muscular power in older adults. Journal of Aging Research, London, v. 1, n. 1, p. 386-387, 2012.

HOGREL, J. Y. Grip strength measured by high precision dynamometry in healthy subjects from 5 to 80 years. BMC Musculoskeletal Disorders, London, v. 16, n. 1, p. 139, 2015.

IOP, R. R. et al. Capacidade de produção de força de preensão isométrica máxima em mulheres com artrite reumatoide: um estudo piloto. Fisioterapia e Pesquisa, São Paulo, v. 22, n. 1, p. 11-16, 2015.

JORGE, M. S. G.; COMIN, J. D. P.; WIBELINGER, L. M. Intervenção fisioterapêutica em um indivíduo com artrite reumatoide, lúpus eritematoso sistêmico e Síndrome de Sjögren: relato de caso. Revista de Ciências Médicas e Biológicas, Salvador, v. 15, n. 2, p. 231237, 2016.

JORGE, M. S. G. et al. Atuação fisioterapêutica em um indivíduo com lúpus eritematoso sistêmico associado à artrite reumatoide e à fibromialgia. ABCS Health Sciences, Santo André, v. 42, n. 1, p. 60-64, 2017.

JORGE, M. S. G.; SCHNORNBERGER, C. M.; SANTOS, T. Intervenção fisioterapêutica nos distúrbios do tecido conjuntivo. In: WIBELINGER, L. M. Fundamentos em

Reabilitação - Disfunções Músculo-Esqueléticas: Prevenção e Reabilitação. 5 ed. Passo Fundo: Saluz; 2017. p. 161-76.

KALUGA, E. et al. Tactile sensitivity on the hands skin in rheumatic patients. Postępy Dermatologii i Alergologii, Poznán, v. 31, n. 3, p. 139-145, 2014.

KAYSER, C.; CORRÊA, M. J. U.; ANDRADE, L. E. C. Fenômeno de Raynaud. Revista Brasileira de Reumatologia, Campinas, v. 49, n. 1, p. 48-63, 2009.

KISNER, C.; COLBY, L. A.; CAROMANO, F. A. Exercícios terapêuticos: fundamentos e técnicas. 5ed. Barueri: Manole, 2009.

LEE, H. J. et al. The impact of digit-related radiographic osteoarthritis of the hand on grip- 
strength and upper extremity disability. Clinical Orthopaedics and Related Research, Philadelphia, v. 470, n. 8, p. 2202-2028, 2012.

MADDALI BONGI, S.; DEL ROSSO, A. How to prescribe physical exercise in rheumatology. Reumatismo, Milão, v. 62, n. 1, p. 4-11, 2010.

MACHADO, R. I. L.; SOUTO, L. M.; FREIRE, E. A. M. Tradução, adaptação cultural e validação para a língua portuguesa (Brasil) do Systemic Sclerosis Questionnaire (SySQ). Revista Brasileira de Reumatologia, Campinas, v. 54, n. 1, p. 95-101, 2014.

MYRA, R. S. et al. Força de preensão palmar em um indivíduo portador de lúpus eritematoso sistêmico e artrite reumatóide: um estudo de caso. Lecturas Educación Física y Deportes, Buenos Aires, v. 20, n. 209, p. 1, 2015.

NOVAES, R. D. et al. Equações de referência para a predição da força de preensão manual em brasileiros de meia idade e idosos. Fisioterapia e Pesquisa, São Paulo, v. 16, n. 3, p. 217-222, 2009.

NUNES, P. M. et al. Relationship between hand function and grip force control in women with hand osteoarthritis. Journal of Rehabilitation Research and Development, Washington, v. 49, n. 6, p. 855-865, 2012.

ØSTERÅS, N.; KJEKEN, I. A tailored hand exercise program improves function of the rheumatoid hand. Journal of Physiotherapy, Hawthorn, v. 61, n. 2, p. 96, 2015.

PORTUGAL. Programa Nacional contra as Doenças Reumáticas. 2013. Disponível em: http://www.myos.pt/downloads/circularpncdoencasreumaticas.pdf. Acesso em: 31 jun. 2017.

SAMPAIO-BARROS, M. M. et al. Low vitamin D serum levels in diffuse systemic sclerosis: a correlation with worst quality of life and severe capillaroscopic findings. Revista Brasileira de Reumatologia, Campinas, v. 56, n. 4, p. 337-344, 2016.

SOCIEDADE BRASILEIRA DE REUMATOLOGIA. Cartilha Lúpus. p. 1-21, 2011.

STOCKTON, K. A. et al. Test-retest reliability of hand-held dynamometry and functional tests in systemic lupus erithematosus. Lupus, Houndmills, v. 20, n. 2, p. 144-150, 2011.

VIANNA, R.; SIMÕES, M. J.; INFORZATO, H. C. B. Lúpus Eritrematoso Sistêmico.

Revista Ceciliana, Santos, v. 2, n. 1, p. 1-3, 2010.

VILLAFAÑE, J. H. et al. Reliability of handgrip strength test in elderly subjects with unilateral thumb carpometacarpal osteoarthritis. Hand (NY), New York, v. 10, n. 2, p. 205209, 2015.

WILLIAMS, M. A. et al. Strengthening And stretching for Rheumatoid Arthritis of the Hand (SARAH). A randomised controlled trial and economic evaluation. Health Technology Assessment, Winchester, v. 19, n. 19, p. 1-222, 2015. 